\title{
Patients with mild cognitive impairment diagnosed at dementia clinic display decreased maximum occlusal force: a cross-sectional study
}

\author{
Hiroyuki Suzuki', Junichi Furuya ${ }^{2,3 *}$, Rena Hidaka ${ }^{4}$, Saki Miyajima ${ }^{4}$, Chiaki Matsubara', Gaku Ohwada ${ }^{5}$, \\ Takashi Asada ${ }^{6}$, Chihiro Akazawa7, Yuji Sato ${ }^{2}$, Haruka Tohara ${ }^{3}$ and Shunsuke Minakuchi ${ }^{1}$
}

\begin{abstract}
Background: Previous research indicates that patients with mild cognitive impairment $(\mathrm{MCl})$ are more likely to have poor oral health and impairments in oral functions, which may be due to few remaining teeth and impaired tongue and lip motor function. However, the oral health of those patients following comprehensive cognitive assessment by a dementia specialist has not been sufficiently investigated. Therefore, this study aimed to clarify the oral function of patients with $\mathrm{MCl}$ and the association between oral health and lower cognitive function.

Methods: This cross-sectional study included 96 participants (men: 35; women: 61; mean age: $73.3 \pm 8.5$ years) who visited a dementia clinic between December 2017 and January 2020. Participants' cognitive function was assessed by a dementia specialist using neuropsychological and hematological tests and neuroimaging immediately after enrollment. The participants were divided into the healthy and $\mathrm{MCl}$ groups according to comprehensive cognitive assessment. Participants'age, sex, body mass index, primary disease, education level, drinking habits, smoking habits, living environment, employment status, and exercise habits were evaluated. Moreover, oral outcomes, including the number of existing teeth, number of functional teeth (natural and prosthetic teeth which were occluded with antagonists), denture use, oral dryness, tongue and lip motor function, tongue pressure, occlusal force, masticatory ability, and swallowing ability were recorded. The Mann-Whitney $U$ test, $x^{2}$, and Fisher's exact tests were used for between-group comparisons. Furthermore, logistic regression analysis using $\mathrm{MCl}$ diagnosis as the target variable was performed.
\end{abstract}

Results: A comprehensive evaluation of the cognitive function of the study participants by the dementia specialist revealed that 48 participants (mean age: $69.8 \pm 8.8$ years) were healthy and 48 (mean age: $76.9 \pm 6.7$ years) had $\mathrm{MCl}$. MCl participants were significantly older $(p<0.001)$ and had significantly fewer existing teeth $(p=0.031)$ and lower maximum occlusal force ( $p=0.019$ ) than healthy participants. Age (odds ratio: 1.126, $p=0.002$ ) and maximum occlusal force (odds ratio: $0.978, p=0.048$ ) were significantly associated with lower cognitive function.

Conclusions: Patients with $\mathrm{MCl}$ had poorer oral health than healthy individuals. Decreased maximum occlusal force was independently associated with lower cognitive function, even when adjusted for age and sex.

Keywords: Mild cognitive impairment, Oral health, Oral status, Oral function, Denture, Maximum occlusal force

*Correspondence: furuyajunichi@gmail.com

${ }^{2}$ Department of Geriatric Dentistry, Showa University School of Dentistry,

2-1-1 Kitasenzoku, Ohta-ku, Tokyo 145-8515, Japan

Full list of author information is available at the end of the article

\section{Background}

With the increasing age of the population globally, the incidence of dementia is also increasing. According to the 2019 World Health Organization [1] guidelines for 
dementia, the global population of patients with dementia is approximately 50 million and is increasing by approximately 10 million each year and is expected to reach 152 million by 2050. Similarly, as the number of dementia patients is increasing rapidly in Japan [2], the management of the oral health of older patients with dementia is becoming an increasingly important issue in dentistry [3]. Older adults with dementia are known to have poor oral health, including poor oral hygiene due to inadequate cleaning of teeth and dentures $[4,5]$, tooth loss due to caries and periodontal disease [6,7], decreased masticatory ability, difficulties in using dentures [8], and impaired swallowing function [9]. This poor oral health leads to loss of pleasure from eating and impaired quality of life, which increases the risk of undernutrition [10] and aspiration pneumonia [11] along with reduced diversity of meals. Therefore, specialized dental interventions for maintaining good oral health in older adults with dementia are vital [12]. However, implementing this is difficult in many situations [13], such as cases of advanced dementia, due to poor cooperation with dental treatments. Reportedly, various oral factors, such as decreased oral hygiene, periodontal disease, fewer remaining teeth, no denture use, and reduced masticatory ability, are related to cognitive decline [14-17]. As such, oral health should be adequately managed before the onset of dementia.

Mild cognitive impairment (MCI) reflects a state in which a person has normal functioning in activities of daily living and general cognition despite the presence of memory impairment that cannot be explained by age and level of education alone [18]. While $14.4 \%-55.6 \%$ of patients with $\mathrm{MCI}$ regain normal cognitive function, the incidence of MCI increases with age, and MCI transitions to dementia in $14.9 \%$ of patients diagnosed after the age of 65 [19]. Therefore, in recent years, MCI has been recognized as a stage preceding dementia. At this stage, in which the patient has intact functioning in activities of daily living and general cognition, active oral management including prosthetic treatment can be performed to maintain oral health. In addition, oral health guidance helps maintain and improve cognitive function in older adults with MCI [20]. Therefore, oral health management is critical to maintaining both cognitive and oral functions. Previous research indicates that patients with MCI are more likely to have poor oral health and impairments in oral functions, such as oral diadochokinesis (ODK), which may be due to few remaining teeth and impaired tongue and lip motor function [21-23]. Furthermore, Mizutani et al. [24] conducted a 2-year longitudinal study of older adults without dementia and reported that an increased risk of MCI, as assessed by MCI biomarkers, was associated with decreased masticatory ability. These findings suggest that oral health management by dental professionals is important for patients with MCI. However, MCI was diagnosed in previous studies based solely on the results of a single neuropsychological test, such as the mini-mental state examination (MMSE) [25] or the Japanese version of the Montreal Cognitive Assessment (MoCA-J) [26]. Ideally, diagnosis should be made by a dementia specialist through a comprehensive evaluation of a patient's cognitive function using hematological [27] and neuropsychological tests, alongside neuroimaging $[28,29]$. However, the oral health of the patients diagnosed with $\mathrm{MCI}$ in this manner has not been elucidated yet.

Therefore, this cross-sectional study aimed to examine the oral function, in particular oral dryness, tongue and lip motor function, tongue pressure, occlusal force, masticatory ability, and swallowing ability, which are parts of the diagnostic criteria for oral hypofunction [30] of patients diagnosed with MCI by a dementia specialist and to determine the association between oral health and lower cognitive function.

\section{Methods \\ Participants}

This cross-sectional study enrolled 111 patients and their family members who visited a dementia clinic with a chief complaint of cognitive decline between December 2017 and January 2020. Family members of the patients were included as participants to minimize the bias of the living environment among the participants as much as possible. The exclusion criteria were as follows: patients who were living in long-term care facilities, patients aged $<50$ years, patients who had been diagnosed with dementia, and patients who were unable to undergo an oral function test. Therefore, two patients younger than 50 years, 12 patients diagnosed with dementia, and one patient who was unable to undergo oral function tests were excluded from this study. Finally, the present cross-sectional study included 96 participants. Participants were diagnosed as healthy or having MCI by a dementia specialist through a comprehensive evaluation using neuropsychological tests such as the MMSE [25]; neuroimaging such as electroencephalography, magnetic resonance imaging, cerebral perfusion scintigraphy, and single-photon emission computed tomography; and hematological tests. The general criteria for MCI diagnosis as reported by Winblad et al. were as follows: a person who was judged as neither normal nor demented based on the diagnostic criteria for dementia such as those in the International Classification of Diseases 10 (ICD-10); a person whose functional activities were mainly preserved or minimally impaired; a person with evidence of cognitive decline measured either by self and/or reported by an informant in conjunction 
with deficits in objective cognitive tasks and/or evidence of decline over time in objective neuropsychological tests [28]. In this study, the diagnostic criteria for MCI were based on these general criteria for MCI. The participants were divided into the healthy and the MCI groups based on a diagnosis of comprehensive cognitive evaluation by a dementia specialist. All participants provided informed consent before enrollment after being given detailed oral and written explanations about the study. This study was approved by the Tokyo Medical and Dental University Faculty of Medicine Ethics Committee (Approval No.: M2017-112).

\section{Measurements}

\section{Participants' basic information}

Participants' age, sex, body mass index (BMI), primary disease, education level, drinking habits, smoking habits, living environment, employment status, and exercise habits were evaluated. Education level, which was classified into the following categories: four-year university graduate, junior college graduate, specialist school graduate, high school graduate, middle school graduate, or other, was used to evaluate each participant's years of education. This was calculated according to the years of study each category required. Living environment was categorized as either living alone or living with family. Drinking habits, smoking habits, employment status, and exercise habits were categorized as either "Yes" or "No."

\section{Oral health assessment}

Oral function was assessed by five dentists and dental hygienists sufficiently trained and proficient in the measurement techniques. Through oral examination, the number of existing teeth, number of functional teeth, and status of denture usage were evaluated. The number of existing teeth was assessed by counting the number of natural teeth, whereas the number of functional teeth which were occluded with antagonists, included prosthetic teeth, such as dentures and implants. Residual roots were excluded from both counts. The number of existing and functional teeth ranged from 0 to 28 , respectively. In terms of the status of denture usage, each participant was classified as either using or not using dentures. Additionally, the following oral functions were evaluated. Denture users underwent all tests while wearing dentures.

\section{Oral dryness}

Oral dryness was evaluated by measuring the wetness at the center of the tongue base using an oral moisturechecking device (Mucus ${ }^{\circledR}$, LIFE Corp., Saitama, Japan) and calculating the median value of three measurements
[31]. Oral dryness was defined as a value of less than 27.0 [30].

\section{Tongue and lip motor function}

ODK while making /pa/ and / ta/ sounds was assessed to evaluate tongue and lip motor function. Participants were instructed to pronounce the syllables $/ \mathrm{pa} /$ and $/ \mathrm{ta} /$ as many times as possible in $5 \mathrm{~s}$, and the number of syllables per second was measured using an automated measuring device (KENKOUKUN Handy, Takei Scientific Instruments Co. Ltd., Niigata, Japan) [32]. Measurements were carried out twice for each syllable, and the maximum value was regarded as the tongue and lip motor function. Reduced tongue and lip motor function were defined as the pronunciation of / $\mathrm{pa} /$ and $/ \mathrm{ta} /$ syllables at a rate of fewer than six times per second [30].

\section{Tongue pressure}

Tongue pressure was measured using a tongue pressure measurement device (TPM-01, JMS, Hiroshima, Japan). The pressure generated when the balloon of the tongue pressure probe was pressed between the tongue and the palate for approximately $5 \mathrm{~s}$ was regarded as tongue pressure [33]. This measurement was performed twice with a 30-s break between measurements, and the maximum value measured was considered the maximum tongue pressure. Low tongue pressure was defined as a maximum tongue pressure of less than $30 \mathrm{kPa}$ [30].

\section{Occlusal force}

Occlusal force was evaluated using a pressure-sensitive sheet (Dental Prescale, Fuji Film Co., Tokyo, Japan) and an analyzer (Occluzer FPD709, Fuji Film Co., Tokyo, Japan), by measuring the maximum occlusal force at the intercuspal position during clenching for $3 \mathrm{~s}$. The pressure-sensitive sheet was inserted into the participant's oral cavity, and the participant was instructed to clench down with maximum force at the intercuspal position for $3 \mathrm{~s}$. The maximum occlusal force was calculated by using the analyzer to remove readings (noise) from the region of the pressure-sensitive sheet that was not in the region that the analyzer indicated deviated from the dentition [34]. Reduced occlusal force was defined as a maximum occlusal force of less than $200 \mathrm{~N}$ [30].

\section{Masticatory ability}

Masticatory ability was evaluated using masticatory ability evaluation gum (Xylitol Mastic Gum, Lotte, Tokyo, Japan) that changes color from green to red the more it is chewed [35]. Participants were instructed to chew the gum 60 times at a rate of once per second, and their masticatory ability was evaluated in terms of change in gum color using the visual scoring method with a 10-stage 
color scale [36]. Reduced masticatory ability was defined as a mastication score of less than six on the color scale [17].

\section{Swallowing ability}

Swallowing ability was evaluated using the modified water swallowing test (MWST) [37]. Participants were instructed to swallow $3 \mathrm{~mL}$ of cold water and their swallowing ability was classified into one of five categories according to the success and condition of swallowing: (1) unable to swallow with choking and/or breathing changes, (2) able to swallow, but with breathing changes, (3) able to swallow with normal breathing, but with choking and/or wet hoarseness, (4) able to swallow with normal breathing and without choking, and (5) in addition to that mentioned for category 4 , able to perform twice repetitive swallowing within $30 \mathrm{~s}$. Reduced swallowing ability was defined as an MWST score of $<3$.

\section{Statistical analysis}

The Mann-Whitney $U$ test was used to compare the healthy and MCI groups in terms of continuous variables, which included age, BMI, years of education, MMSE, number of existing teeth, number of functional teeth, oral dryness, ODK $/ \mathrm{pa} /$, ODK $/ \mathrm{ta} /$, maximum tongue pressure, maximum occlusal force, masticatory ability, and swallowing ability. Categorical variables, such as sex and the proportion of participants in each group with reduced oral function were compared using $x^{2}$ and Fisher's exact tests. Furthermore, to determine oral function related to lower cognitive function when adjusted for age and sex, a logistic regression analysis was performed using MCI diagnosis as the target variable (healthy $=0$, $\mathrm{MCI}=1$ ) and age, sex, oral dryness, ODK /pa/, ODK / $\mathrm{ta} /$, maximum tongue pressure, maximum occlusal force, masticatory ability, and swallowing ability as independent variables. Statistical analyses were performed using SPSS Ver. 27 (IBM Japan, Tokyo, Japan), with a significance level of $5 \%$.

\section{Results}

\section{Participants' characteristics}

A comprehensive evaluation of the cognitive function of the study participants (men: 35; women: 61; mean age: $73.3 \pm 8.5$ years) by the dementia specialist revealed that 48 participants (mean age: $69.8 \pm 8.8$ years) were healthy and 48 participants (mean age: $76.9 \pm 6.7$ years) had MCI. Table 1 represents the basic information of the participants in the healthy and MCI groups. Participants in the MCI group were significantly older $(\mathrm{p}<0.001)$ and had significantly lower MMSE $(\mathrm{p}<0.001)$ than the participants in the healthy group. Although there were significant between-group differences in employment status $(\mathrm{p}=0.038)$ and exercise habits $(\mathrm{p}=0.030)$, there were no significant differences between the groups in terms of the other basic information.

\section{Oral function}

Table 2 represents the oral function results of participants in the healthy and MCI groups. Although the MCI group had significantly fewer existing teeth $(\mathrm{p}=0.031)$ than the healthy group, the fact that denture use was significantly more common in the MCI group $(\mathrm{p}=0.025)$ indicated there was no significant difference between the two groups in terms of the number of functional teeth. Furthermore, the maximum occlusal force was significantly lower in the MCI group $(p=0.019)$. When assessed based on the diagnostic criteria of oral hypofunction [29], the median values of the parameters of oral function were higher for both groups; however, the MCI group had a significantly higher proportion of participants with impaired ODK $/ \mathrm{pa} /$ than the healthy group $(\mathrm{p}=0.036)$.

\section{Association between oral function and lower cognitive function}

Table 3 represents the results of logistic regression analysis. Even after adjusting for age (odds ratio: 1.126, 95\% confidence interval: $1.045-1.212, \mathrm{p}=0.002)$ and sex (odds ratio: 0.528, 95\% confidence interval: 0.196-1.423, $\mathrm{p}=0.207$ ), maximum occlusal force (odds ratio: 0.978 , 95\% confidence interval: $0.956-1.000, \mathrm{p}=0.048)$ was significantly associated with lower cognitive function.

\section{Discussion}

The findings of this study revealed that patients with MCI diagnosed by the dementia specialist were older and tended to participate in social circumstances, such as employment or exercise, less frequently than healthy individuals. Furthermore, despite maintained oral function, patients with MCI tended to display reduced maximum occlusal force. Moreover, from the viewpoint of oral hypofunction [30], a high proportion of patients with MCI presented with reduced tongue and lip motor function. Although oral function decreased by aging [38], a reduction in the maximum occlusal force, even when adjusted for age and sex, was independently associated with lower cognitive function. Therefore, the findings of this study suggest that implementing appropriate dental interventions without overlooking subtle changes in oral function such as occlusal force and working with other specialists, such as dementia specialists, might help prevent cognitive decline and the onset of dementia.

Petersen et al. reported that the incidence of MCI increases with age; it is $6.7 \%$ between 60 and 64 years, $8.4 \%$ between 65 and 69 years, $10.1 \%$ between 70 and 
Table 1 Participants' basic information

\begin{tabular}{|c|c|c|c|c|c|c|c|c|c|}
\hline & \multicolumn{4}{|l|}{ Healthy } & \multicolumn{4}{|l|}{$\mathrm{MCl}$} & \multirow[t]{2}{*}{$p$ value } \\
\hline & Mean \pm SD & Median & $\mathbf{n}$ & $\%$ & Mean \pm SD & Median & $\mathbf{n}$ & $\%$ & \\
\hline Age & $69.8 \pm 8.8$ & 71.5 & 48 & & $76.9 \pm 6.7$ & 78 & 48 & & $<0.001^{*}$ \\
\hline \multicolumn{10}{|l|}{ Sex } \\
\hline Male & & & 14 & 29.2 & & & 21 & 43.8 & 0.138 \\
\hline Female & & & 34 & 70.8 & & & 27 & 56.2 & \\
\hline BMI $\left(\mathrm{kg} / \mathrm{m}^{2}\right)$ & $22.5 \pm 10.7$ & 22.1 & 48 & & $22.7 \pm 4.0$ & 22.4 & 48 & & 0.921 \\
\hline \multicolumn{10}{|l|}{ Primary disease } \\
\hline Hypertension & & & 16 & 33.3 & & & 24 & 50.0 & 0.098 \\
\hline Cerebrovascular disease & & & 2 & 4.2 & & & 4 & 8.3 & 0.399 \\
\hline Heart disease & & & 3 & 6.3 & & & 6 & 12.5 & 0.294 \\
\hline Diabetes mellitus & & & 5 & 10.4 & & & 5 & 10.4 & 1.000 \\
\hline Hyperlipidemia & & & 10 & 20.8 & & & 9 & 18.8 & 0.798 \\
\hline Malignant tumor & & & 3 & 6.3 & & & 7 & 14.6 & 0.181 \\
\hline Depression & & & 2 & 4.2 & & & 1 & 2.1 & 0.557 \\
\hline Neurologic disease & & & 0 & 0.0 & & & 3 & 6.3 & 0.078 \\
\hline Others & & & 17 & 35.4 & & & 24 & 50.0 & 0.149 \\
\hline Years of education & $14.3 \pm 1.8$ & 14 & 48 & & $13.8 \pm 2.5$ & 14 & 48 & & 0.600 \\
\hline \multicolumn{10}{|l|}{ Alcohol } \\
\hline Yes & & & 22 & 45.8 & & & 22 & 45.8 & 1.00 \\
\hline No & & & 26 & 54.2 & & & 26 & 54.2 & \\
\hline \multicolumn{10}{|l|}{ Smoking } \\
\hline Yes & & & 2 & 4.2 & & & 4 & 8.3 & 0.677 \\
\hline No & & & 46 & 95.8 & & & 44 & 91.7 & \\
\hline \multicolumn{10}{|l|}{ Living environment } \\
\hline Alone & & & 3 & 6.3 & & & 9 & 18.8 & 0.064 \\
\hline With family & & & 45 & 93.7 & & & 38 & 79.2 & \\
\hline \multicolumn{10}{|l|}{ Employment } \\
\hline Yes & & & 25 & 52.1 & & & 15 & 31.3 & $0.038^{*}$ \\
\hline No & & & 23 & 47.9 & & & 33 & 68.7 & \\
\hline \multicolumn{10}{|l|}{ Exercise habits } \\
\hline Yes & & & 37 & 77.1 & & & 27 & 56.2 & $0.030^{*}$ \\
\hline No & & & 11 & 22.9 & & & 21 & 43.8 & \\
\hline MMSE score & $28.7 \pm 1.2$ & 29 & 47 & & $23.8 \pm 3.0$ & 24 & 48 & & $<0.001^{* *}$ \\
\hline
\end{tabular}

$\mathrm{MCl}$ : mild cognitive impairment, SD: standard deviation, BMI: body mass index, MMSE: mini-mental state examination ${ }^{*} \mathrm{p}<0.05$ Healthy group vs $\mathrm{MCl}$ group, Chi-square test

${ }^{* *} \mathrm{p}<0.05$ Healthy group vs $\mathrm{MCl}$ group, Mann-Whitney $U$ test

74 years, $14.8 \%$ between 75 and 79 years, and $25.2 \%$ between 80 and 84 years of age [19]. Previous studies have revealed that reduced social participation, which includes activities, such as working [39] and exercise [40] are related to cognitive decline. The participants in the MCI group in this study were also significantly older than those in the healthy group, and the fact that many of them were not working or exercising revealed a trend similar to that discussed in previous reports [39, 40]. Furthermore, the median MMSE score of MCI patients in this study was 24: lower than the cut-off MMSE score
[41] for MCI diagnosis. This may be due to the participants in this study having been diagnosed with $\mathrm{MCI}$ based on a comprehensive evaluation using neuropsychological tests, such as the MMSE [24], in addition to neuroimaging and hematological tests and because many of them were patients who sought medical attention at a dementia clinic.

Egashira et al. [42] compared the oral function and environment of participants classified into healthy and MCI groups based on MoCA-J scores. They reported that the participants in the MCI group were significantly 
Table 2 Oral status of the participants in the healthy and $\mathrm{MCl}$ groups

\begin{tabular}{|c|c|c|c|c|c|c|c|c|c|}
\hline & \multicolumn{4}{|l|}{ Healthy } & \multicolumn{4}{|l|}{$\mathrm{MCl}$} & \multirow[t]{2}{*}{$\mathrm{p}$ value } \\
\hline & Mean \pm SD & Median & $\mathbf{n}$ & $\%$ & Mean \pm SD & Median & $\mathrm{n}$ & $\%$ & \\
\hline Number of existing teeth & $24.9 \pm 5.6$ & 27 & 48 & & $20.2 \pm 9.8$ & 24.5 & 48 & & $0.031^{*}$ \\
\hline Number of functional teeth & $27.5 \pm 1.8$ & 28 & 48 & & $27.4 \pm 2.0$ & 28 & 48 & & 0.865 \\
\hline \multicolumn{10}{|l|}{ Denture use } \\
\hline Yes & & & 9 & 18.8 & & & 19 & 39.6 & $0.025^{* *}$ \\
\hline No & & & 39 & 81.2 & & & 29 & 60.4 & \\
\hline Oral dryness & $29.7 \pm 3.9$ & 30.6 & 48 & & $29.4 \pm 3.0$ & 30.1 & 48 & & 0.371 \\
\hline Maintained & & & 43 & 89.6 & & & 39 & 81.2 & 0.247 \\
\hline Reduced & & & 5 & 10.4 & & & 9 & 18.8 & \\
\hline ODK/pa/ & $6.6 \pm 0.6$ & 6.8 & 48 & & $6.5 \pm 0.9$ & 6.6 & 48 & & 0.586 \\
\hline Maintained & & & 43 & 89.6 & & & 35 & 72.9 & $0.036^{* *}$ \\
\hline Reduced & & & 5 & 10.4 & & & 13 & 27.1 & \\
\hline ODK/ta/ & $6.7 \pm 0.7$ & 6.6 & 48 & & $6.4 \pm 0.8$ & 6.2 & 48 & & 0.086 \\
\hline Maintained & & & 42 & 87.5 & & & 35 & 72.9 & 0.073 \\
\hline Reduced & & & 6 & 12.5 & & & 13 & 27.1 & \\
\hline Tongue pressure & $33.3 \pm 8.1$ & 32.3 & 48 & & $33.3 \pm 7.4$ & 33.3 & 48 & & 0.878 \\
\hline Maintained & & & 33 & 68.7 & & & 35 & 72.9 & 0.653 \\
\hline Reduced & & & 15 & 31.3 & & & 13 & 27.1 & \\
\hline Maximum occlusal force (/10 N) & $41.1 \pm 23.4$ & 38.1 & 48 & & $30.2 \pm 21.0$ & 27.9 & 48 & & $0.019^{*}$ \\
\hline Maintained & & & 38 & 79.2 & & & 31 & 64.6 & 0.112 \\
\hline Reduced & & & 10 & 20.8 & & & 17 & 35.4 & \\
\hline Masticatory ability & $6.3 \pm 1.7$ & 7 & 48 & & $6.0 \pm 1.7$ & 6 & 48 & & 0.305 \\
\hline Maintained & & & 36 & 75.0 & & & 30 & 62.5 & 0.186 \\
\hline Reduced & & & 12 & 25.0 & & & 18 & 37.5 & \\
\hline Swallowing ability & $5.0 \pm 0.3$ & 5 & 48 & & $4.9 \pm 0.3$ & 5 & 48 & & 0.320 \\
\hline Maintained & & & 47 & 97.9 & & & 47 & 97.9 & 1.000 \\
\hline Reduced & & & 1 & 2.1 & & & 1 & 2.1 & \\
\hline
\end{tabular}

MCl: mild cognitive impairment, SD: standard deviation, ODK: oral diadochokinesis ${ }^{*} \mathrm{p}<0.05$ Healthy group vs MCI group, Mann-Whitney $\mathrm{U}$ test

${ }^{* *} \mathrm{p}<0.05$ Healthy group vs $\mathrm{MCl}$ group, Chi-square test

Table 3 Results of logistic regression analysis with $\mathrm{MCl}$ diagnosis as the dependent variable (Healthy $=0, \mathrm{MCl}=1$ )

\begin{tabular}{llll}
\hline Independent variables & Odds ratio & $\begin{array}{l}\mathbf{9 5 \%} \\
\text { Confidence } \\
\text { interval }\end{array}$ & p value \\
\hline Age & 1.126 & $1.045-1.212$ & $0.002^{*}$ \\
Sex & 0.528 & $0.196-1.423$ & 0.207 \\
Oral dryness & 0.979 & $0.856-1.121$ & 0.762 \\
ODK/pa/ & 1.297 & $0.473-3.554$ & 0.613 \\
ODK/ta/ & 0.710 & $0.235-2.149$ & 0.545 \\
Tongue pressure & 1.027 & $0.962-1.096$ & 0.420 \\
Maximum occlusal force & 0.978 & $0.956-1.000$ & $0.048^{*}$ \\
Masticatory ability & 1.142 & $0.831-1.568$ & 0.413 \\
Swallowing ability & 0.896 & $0.215-3.746$ & 0.881 \\
\hline
\end{tabular}

Sex: Male $=0$, Female $=1$. All other independent variables were continuous variables. Maximum occlusal force shown per 10 Newton

$\mathrm{MCl}$ : mild cognitive impairment, ODK: oral diadochokinesis

${ }^{*} p<0.05$, Logistic regression analysis older than those in the healthy group and that the MCI group had significantly fewer existing teeth, significantly lower masticatory ability, and significantly lower maximum tongue pressure than the healthy group. Further, the lower number of teeth and tongue pressure had a significant impact on the decline in cognitive function, even after adjusting for age. Similarly, in our study the maximum occlusal force of the participants in the MCI group was significantly reduced compared to the healthy group, and, even when adjusted for age and sex, this decrease in maximum occlusal force was significantly associated with lower cognitive function. In a recent study, oral function was demonstrated to decline with age [38]. Therefore, reduced oral function in the MCI group observed in a previous study [42] and the present study may be due to age-related effects. However, both studies reported that poor oral function was independently associated with lower cognitive function, even when adjusted for age 
or sex, which suggests that oral function may decline in MCI patients regardless of age.

The oral function of participants with MCI was relatively maintained in the context of the diagnostic criteria for oral hypofunction [30]. These results suggest that, while patients with MCI have reduced oral function compared to healthy individuals, this reduction may be mild. Conversely, the percentage of participants with reduced $\mathrm{ODK} / \mathrm{pa} /$ was higher in this study than among normal participants, which might be related to the fact that the participants in this study did not participate in social activities, such as employment. Nevertheless, since previous reports suggest that such a decline in oral function may lead to early detection of MCI [21], that a decrease in tongue pressure and ODK are associated with a decreased MMSE score, and that a decrease in maximum occlusal force is associated with a decrease in the MoCAJ score [43], it is important to provide appropriate dental intervention without overlooking subtle changes in oral function for prevention or early detection of dementia. This is an important responsibility of dental practitioners in a super-aged society. In particular, according to Matsubara et al. [20], older adults with MCI who received personalized dental health guidance displayed significant improvement in cognitive function compared to older adults with MCI who did not receive such guidance. Therefore, initiating dental check-ups before MCI onset and appropriately managing oral function in the event of MCI development may be important for maintaining and improving the oral and cognitive functions of older adults with MCI.

This study has several limitations. The first is the potential selection bias that arises from recruiting participants who voluntarily visited a dementia clinic. Since the participants in this study had a relatively high level of health literacy and many were using dentures for missing teeth, they were also considered to have a relatively high level of interest in oral health. Therefore, our findings may not reflect the reality of oral health in all patients with MCI. Additionally, there are some differences in age and other characteristics between the healthy group and the $\mathrm{MCI}$ group in this study. A large-scale investigation of community-dwelling older adults, adjusting for background factors where possible, is necessary to elucidate the oral health of patients with MCI. However, it is difficult to perform comprehensive cognitive function tests conducted by a dementia specialist, as done in this study, in all participants of a large-scale investigation. In fact, most previous large-scale studies [21-23] have diagnosed MCI solely based on neuropsychological test results. Neuropsychological tests allow for the easy screening of cognitive function, and while their reliability and validity have been studied [41], some reports indicate that it is difficult to accurately diagnose MCI based solely on neuropsychological test results [44]. As such, in this study, the oral health of individuals who had undergone comprehensive cognitive testing at a dementia clinic and had been diagnosed with MCI by a dementia specialist were examined. Second, oral hygiene, which was one of the diagnostic criteria for oral hypofunction, was not evaluated because the purpose of this study was to examine the kind of oral function that is related to lower cognitive function. In addition, evaluation of chewing and swallowing abilities was done using a different method rather than using the diagnostic criteria for oral hypofunction in favor of simplicity of examination. If the evaluation methods for oral hygiene, chewing ability, and swallowing ability according to the diagnostic criteria for oral hypofunction had been used in this study, the actual condition of oral hypofunction in MCI patients could have been evaluated more accurately. In addition, since this study was not conducted in a dental clinic, evaluation of denture adaptation or periodontal parameters such as plaque index and BOP were not performed because it was considered difficult to accurately evaluate the results. Third, MCI patients were classified into amnestic MCI and non-amnestic MCI based on their symptoms, but this classification was not used in this study. This classification is known to be useful in predicting what type of dementia may develop in the future [45]. Therefore, if MCI patients had been classified into amnestic MCI and non-amnestic MCI in this study and then assessed for their oral function, the oral function associated with each dementia type might have been elucidated. Fourth, the logistic regression analysis excluded the number of existing teeth and the status of denture use as independent variables, despite previous reports demonstrating that these two factors impact cognitive function $[15,16]$. However, these two metrics are also known to correlate with occlusal force and masticatory ability [46]; this study confirms this association by indicating significant correlations between the number of existing teeth and maximum occlusal force $(r=0.524, p<0.001$, Spearman's rank correlation coefficient analysis), between the number of existing teeth and masticatory ability $(\mathrm{r}=0.421, \mathrm{p}<0.001$, Spearman's rank correlation coefficient analysis), between the status of denture use ( $\mathrm{Yes}=0, \mathrm{No}=1)$ and maximum occlusal force $(\mathrm{r}=0.412, \mathrm{p}<0.001$, Spearman's rank correlation coefficient analysis), and between the status of denture use $(\mathrm{Yes}=0, \mathrm{No}=1)$ and masticatory ability $(\mathrm{r}=0.367, \mathrm{p}<0.01$, Spearman's rank correlation coefficient analysis). Therefore, to avoid multicollinearity, the number of existing teeth and status of denture use were excluded from independent variables. Moreover, previous research indicates that exercise habits and employment status are associated with cognitive function [39, 
40]. In contrast, the decline in social participation represented by these exercise habits and employment status is also reportedly associated with the decline in oral function $[47,48]$. In light of these reports, in this study, exercise habits and employment status were not included as independent variables in the logistic regression analysis. Finally, as this was a cross-sectional study conducted at a single center, it could not elucidate the causal relationship between cognitive and oral health changes. Therefore, longitudinal studies are warranted to elucidate the relationship between oral health and cognitive decline in patients with $\mathrm{MCI}$, and interventional studies are needed to determine the most effective oral health intervention to prevent cognitive decline. Moreover, it is extremely important to clarify the cut-off values of oral function that can be used as a reference for MCI diagnosis, and a large-scale survey of MCI patients based on a comprehensive cognitive function assessment is warranted.

\section{Conclusions}

In conclusion, the oral health of patients with MCI diagnosed by the dementia specialist was slightly poorer than that of healthy individuals. In particular, a decrease in the maximum occlusal force was independently associated with lower cognitive function, even when adjusted for age and sex. The findings of this study suggest that for prevention of cognitive decline, it is important to implement appropriate oral management without overlooking even the most subtle changes in oral health.

\section{Abbreviations \\ MCI: Mild cognitive impairment; BMI: Body mass index; ODK: Oral diadochoki- nesis; MMSE: Mini Mental State Examination; MoCA-J: Japanese version of the} Montreal Cognitive Assessment; MWST: Modified water swallowing test.

\section{Acknowledgements}

The authors specially thank all staff members and participants of the study

\section{Authors' contributions}

Conceptualization, JF, TA, CA, HT, and SM; data collection, $\mathrm{HS}, \mathrm{RH}, \mathrm{SM}$, and $\mathrm{CM}$; Formal analysis, $\mathrm{HS}, \mathrm{RH}$, and $\mathrm{CM}$; Funding acquisition, GO; writing —original draft preparation, $\mathrm{HS}$, and JF; writing - review and editing, RH, GO, TA, CA, YS, $H T$, and SM. All authors read and approved the final manuscript.

\section{Funding}

This work was supported by JSPS KAKENHI (Grant Number 18K09654).

\section{Availability of data and materials}

All data generated or analyzed during this study are included in this published article.

\section{Declarations}

\section{Ethics approval and consent to participate}

This study was approved by the Tokyo Medical and Dental University Faculty of Medicine Ethics Committee (Approval No.: M2017-112). All participants provided informed consent.

\section{Consent for publication}

Not applicable.

\section{Competing interests}

The authors declare no conflicts of interest associated with this work. The funders had no role in the design of the study; in the collection, analyses, or interpretation of data; in the writing of the manuscript; or in the decision to publish the results.

\section{Author details}

${ }^{1}$ Gerodontology and Oral Rehabilitation, Department of Gerontology and Gerodontology, Graduate School of Medical and Dental Sciences, Tokyo Medical and Dental University, 1-5-45 Yushima, Bunkyo-ku, Tokyo 113-8549, Japan. ${ }^{2}$ Department of Geriatric Dentistry, Showa University School of Dentistry, 2-1-1 Kitasenzoku, Ohta-ku, Tokyo 145-8515, Japan. ${ }^{3}$ Dysphagia Rehabilitation, Department of Gerontology and Gerodontology, Graduate School of Medical and Dental Sciences, Tokyo Medical and Dental University, 1-5-45 Yushima, Bunkyo-ku, Tokyo 113-8549, Japan. ${ }^{4}$ Department of Oral Health Sciences for Community Welfare, Graduate School of Medical and Dental Sciences, Tokyo Medical and Dental University, 1-5-45 Yushima, Bunkyo-ku, Tokyo 113-8549, Japan. ${ }^{5}$ Ohwada Dental Office, 1-2-45 Honcho, Kuki City, Saitama 346-0005, Japan. ${ }^{6}$ Memory Clinic Ochanomizu, 1-5-34 Yushima, Bunkyo-ku, Tokyo 113-0034, Japan. ${ }^{7}$ Intractable Disease Research Centre, Juntendo University School of Medicine, 2-1-1 Hongo, Bunkyo-ku, Tokyo 113-8421, Japan.

Received: 12 October 2021 Accepted: 15 December 2021

Published online: 27 December 2021

\section{References}

1. World Health Organization. Risk reduction of cognitive decline and dementia 2019. https://apps.who.int/iris/bitstream/handle/10665/ 312180/9789241550543-eng.pdf. Accessed 7 Jan 2021.

2. Ohara T, Hata J, Yoshida D, Mukai N, Nagata M, Iwaki T, et al. Trends in dementia prevalence, incidence, and survival rate in a Japanese community. Neurology. 2017;88:1925-32.

3. Gao SS, Chu CH, Young FYF. Oral health and care for elderly people with Alzheimer's disease. Int J Environ Res Public Health. 2020;17:5713.

4. Gil-Montoya JA, Sánchez-Lara I, Carnero-Pardo C, Fornieles-Rubio F, Montes J, Barrios R, et al. Oral hygiene in the elderly with different degrees of cognitive impairment and dementia. J Am Geriatr Soc. 2017:65:642-7.

5. Jockusch J, Hopfenmüller W, Nitschke I. Influence of cognitive impairment and dementia on oral health and the utilization of dental services: findings of the Oral Health, Bite force and Dementia Study (OrBiD). BMC Oral Health. 2021;21:399.

6. Chen X, Shuman SK, Hodges JS, Gatewood LC, Xu J. Patterns of tooth loss in older adults with and without dementia: a retrospective study based on a Minnesota cohort. J Am Geriatr Soc. 2010;58:2300-7.

7. Syrjälä AM, Ylöstalo P, Ruoppi P, Komulainen K, Hartikainen S, Sulkava R, et al. Dementia and oral health among subjects aged 75 years or older. Gerodontology. 2012;29:36-42.

8. Adam H, Preston AJ. The oral health of individuals with dementia in nursing homes. Gerodontology. 2006;23:99-105.

9. Sato E, Hirano H, Watanabe Y, Edahiro A, Sato K, Yamane G, et al. Detecting signs of dysphagia in patients with Alzheimer's disease with oral feeding in daily life. Geriatr Gerontol Int. 2014;14:549-55.

10. Sadamori S, Hayashi S, Fujihara I, Abekura H, Hamada T, Akagawa Y. Nutritional status and oral status of the elderly with dementia: a 2-year study. Gerodontology. 2012;29:e756-60.

11. Edahiro A, Hirano H, Yamada R, Chiba Y, Watanabe Y, Tonogi M, et al. Factors affecting independence in eating among elderly with Alzheimer's disease. Geriatr Gerontol Int. 2012;12:481-90.

12. Morishita S, Watanabe Y, Ohara Y, Edahiro A, Sato E, Suga T, et al. Factors associated with older adults' need for oral hygiene management by dental professionals. Geriatr Gerontol Int. 2016;16:956-62.

13. Lauritano D, Moreo G, Della Vella F, Di Stasio D, Carinci F, Lucchese A, et al. Oral health status and need for oral care in an aging population: a systematic review. Int J Environ Res Public Health. 2019;16:4558. 
14. Zenthöfer A, Baumgart D, Cabrera T, Rammelsberg P, Schröder J, Corcodel N, et al. Poor dental hygiene and periodontal health in nursing home residents with dementia: an observational study. Odontology. 2017; 105:208-13.

15. Nilsson H, Berglund JS, Renvert S. Periodontitis, tooth loss and cognitive functions among older adults. Clin Oral Investig. 2018;22:2103-9.

16. Yamamoto T, Kondo $K$, Hirai H, Nakade M, Aida J, Hirata Y. Association between self-reported dental health status and onset of dementia: a 4-year prospective cohort study of older Japanese adults from the Aichi Gerontological Evaluation Study (AGES) Project. Psychosom Med. 2012;74:241-8

17. Kimura Y, Ogawa H, Yoshihara A, Yamaga T, Takiguchi T, Wada T, et al. Evaluation of chewing ability and its relationship with activities of daily living, depression, cognitive status and food intake in the communitydwelling elderly. Geriatr Gerontol Int. 2013;13:718-25.

18. Petersen RC, Caracciolo B, Brayne C, Gauthier S, Jelic V, Fratiglioni L. Mild cognitive impairment: a concept in evolution. J Intern Med. 2014;275:214-28.

19. Petersen RC, Lopez O, Armstrong MJ, Getchius TSD, Ganguli M, Gloss D, et al. Practice guideline update summary: mild cognitive impairment: report of the Guideline Development, Dissemination, and Implementation Subcommittee of the American Academy of Neurology. Neurology. 2018;90:126-35.

20. Matsubara C, Shirobe M, Furuya J, Watanabe Y, Motokawa K, Edahiro A, et al. Effect of oral health intervention on cognitive decline in community-dwelling older adults: a randomized controlled trial. Arch Gerontol Geriatr. 2021;92:104267.

21. Watanabe Y, Arai H, Hirano H, Morishita S, Ohara Y, Edahiro A, et al. Oral function as an indexing parameter for mild cognitive impairment in older adults. Geriatr Gerontol Int. 2018;18:790-8.

22. Hatta K, Ikebe $K$, Gondo $Y$, Kamide $K$, Masui $Y$, Inagaki H, et al. Influence of lack of posterior occlusal support on cognitive decline among 80-yearold Japanese people in a 3-year prospective study. Geriatr Gerontol Int. 2018;18:1439-46.

23. Kugimiya Y, Ueda T, Watanabe Y, Takano T, Edahiro A, Awata S, et al. Relationship between mild cognitive decline and oral motor functions in metropolitan community-dwelling older Japanese: the Takashimadaira study. Arch Gerontol Geriatr. 2019;81:53-8.

24. Mizutani S, Egashira R, Yamaguchi M, Tamai K, Yoshida M, Kato T, et al. Changes in oral and cognitive functions among older Japanese dental outpatients: a 2-year follow-up study. J Oral Rehabil. 2021. https://doi.org/ 10.1111/joor.13224.

25. Folstein MF, Folstein SE, McHugh PR. "Mini-mental state". A practical method for grading the cognitive state of patients for the clinician. J Psychiatr Res. 1975;12:189-98.

26. Fujiwara Y, Suzuki H, Yasunaga M, Sugiyama M, ljuin M, Sakuma N, et al. Brief screening tool for mild cognitive impairment in older Japanese: validation of the Japanese version of the Montreal Cognitive Assessment. Geriatr Gerontol Int. 2010;10:225-32.

27. Liu S, Suzuki H, Ito H, Korenaga T, Akatsu H, Meno K, et al. Serum levels of proteins involved in amyloid- $\beta$ clearance are related to cognitive decline and neuroimaging changes in mild cognitive impairment. Alzheimers Dement (Amst). 2019;11:85-97.

28. Winblad B, Palmer K, Kivipelto M, Jelic V, Fratiglioni L, Wahlund LO, et al. Mild cognitive impairment-beyond controversies, towards a consensus: report of the International Working Group on Mild Cognitive Impairment. J Intern Med. 2004;256:240-6.

29. Kasper S, Bancher C, Eckert A, Förstl H, Frölich L, Hort J, et al. Management of mild cognitive impairment $(\mathrm{MCl})$ : the need for national and international guidelines. World J Biol Psychiatry. 2020;21:579-94.

30. Minakuchi S, Tsuga K, Ikebe K, Ueda T, Tamura F, Nagao K, et al. Oral hypofunction in the older population: position paper of the Japanese Society of Gerodontology in 2016. Gerodontology. 2018;35:317-24.

31. Yamada H, Nakagawa Y, Nomura Y, Yamamoto K, Suzuki M, Watanabe NY, et al. Preliminary results of moisture checker for Mucus in diagnosing dry mouth. Oral Dis. 2005;11:405-7.

32. Watanabe $Y$, Hirano H, Arai H, Morishita S, Ohara Y, Edahiro A, et al. Relationship between frailty and oral function in community-dwelling elderly adults. J Am Geriatr Soc. 2017;65:66-76.
33. Tsuga K, Maruyama M, Yoshikawa M, Yoshida M, Akagawa Y. Manometric evaluation of oral function with a hand-held balloon probe. J Oral Rehabil. 2011;38:680-5.

34. Shiga $H$, Komino M, Uesugi $H$, Sano M, Yokoyama M, Nakajima K, et al. Comparison of two dental prescale systems used for the measurement of occlusal force. Odontology. 2020;108:676-80.

35. Hama Y, Kanazawa M, Minakuchi S, Uchida T, Sasaki Y. Properties of a color-changeable chewing gum used to evaluate masticatory performance. J Prosthodont Res. 2014;58:102-6.

36. Kugimiya Y, Watanabe Y, Shirobe M, Motohashi Y, Motokawa K, Edahiro A, et al. A comparison of colorimetric and visual methods for the assessment of masticatory performance with color-changeable chewing gum in older persons. J Dent Sci. 2021;16:380-8.

37. Tohara H, Saitoh E, Mays KA, Kuhlemeier K, Palmer JB. Three tests for predicting aspiration without videofluorography. Dysphagia. 2003;18:126-34.

38. Iyota K, Mizutani S, Oku S, Asao M, Futatsuki T, Inoue R, et al. A cross-sectional study of age-related changes in oral function in healthy japanese individuals. Int J Environ Res Public Health. 2020;17:1376.

39. Krueger KR, Wilson RS, Kamenetsky JM, Barnes LL, Bienias JL, Bennett DA. Social engagement and cognitive function in old age. Exp Aging Res. 2009;35:45-60.

40. Larson EB, Wang L, Bowen JD, McCormick WC, Teri L, Crane P, et al. Exercise is associated with reduced risk for incident dementia among persons 65 years of age and older. Ann Intern Med. 2006;144:73-81.

41. Tsai JC, Chen CW, Chu H, Yang HL, Chung MH, Liao YM, et al. Comparing the sensitivity, specificity, and predictive values of the Montreal Cognitive Assessment and Mini-Mental State Examination when screening people for mild cognitive impairment and dementia in Chinese population. Arch Psychiatr Nurs. 2016;30:486-91.

42. Egashira R, Mizutani S, Yamaguchi M, Kato T, Umezaki Y, Oku S, et al. Low tongue strength and the number of teeth present are associated with cognitive decline in older Japanese dental outpatients: a cross-sectional study. Int J Environ Res Public Health. 2020;17:8700.

43. Ikebe K, Gondo Y, Kamide K, Masui Y, Ishizaki T, Arai Y, et al. Occlusal force is correlated with cognitive function directly as well as indirectly via food intake in community-dwelling older Japanese: from the SONIC study. PLOS ONE. 2018;13:e0190741.

44. Arevalo-Rodriguez I, Smailagic N, Roqué I, Figuls M, Ciapponi A, SanchezPerez E, et al. Mini-Mental State Examination (MMSE) for the detection of Alzheimer's disease and other dementias in people with mild cognitive impairment (MCI). Cochrane Database Syst Rev. 2015;2015:CD010783.

45. Petersen RC, Roberts RO, Knopman DS, Boeve BF, Geda YE, Ivnik RJ, et al. Mild cognitive impairment: ten years later. Arch Neurol. 2009;66:1447-55.

46. Ikebe K, Matsuda K, Kagawa R, Enoki K, Okada T, Yoshida M, et al. Masticatory performance in older subjects with varying degrees of tooth loss. J Dent. 2012;40:71-6.

47. Morishita M, Ikeda T, Saito N, Sanou M, Yasuda M, Takao S. Relationship between oral function and life-space mobility or social networks in community-dwelling older people: a cross-sectional study. Clin Exp Dent Res. 2021;7:552-60.

48. Hasegawa Y, Sakuramoto-Sadakane A, Nagai K, Tamaoka J, Oshitani M, Ono T, et al. Does oral hypofunction promote social withdrawal in the older adults? A longitudinal survey of elderly subjects in rural Japan. Int J Environ Res Public Health. 2020;17:8904.

\section{Publisher's Note}

Springer Nature remains neutral with regard to jurisdictional claims in published maps and institutional affiliations. 